\title{
Prevalence and factors associated with intestinal parasitic infections among food handlers working at higher public University student's cafeterias and public food establishments in Ethiopia: a systematic review and meta-analysis
}

\author{
Birhan Alemnew1', Getnet Gedefaw², Gedefaw Diress ${ }^{3}$ and Asmamaw Demis Bizuneh ${ }^{4 *}$ [D
}

\begin{abstract}
Background: Intestinal infection is still an important public health problem in low-income countries. Food handlers may be infected by a wide range of enteropathogens and have been implicated in the transmission of many infections to the public. Therefore, the aim of this review was to produce the pooled prevalence and factors associated with intestinal parasitic infections among food handlers working at higher public University student's cafeterias and public food establishments in Ethiopia.

Methods: Articles published in PubMed/Medline, Hinari, Web of Science, Science Direct, and Google Scholar were used using a search strategy. Observational studies (cross-sectional) revealing the prevalence and factors associated with intestinal parasitic infections at higher public University student's cafeterias and public food establishments were incorporated. Meta-analysis was computed using STATA version 14 statistical software. Heterogeneity of the study was assessed using Cochrane $\mathrm{Q}$ test statistics and $\mathrm{I}^{2}$ test. The pooled prevalence of the intestinal parasitic infection and associated factors among food handlers was calculated by the random-effect model.

Results: Out of 138 reviewed studies, 18 studies were included to estimate the pooled prevalence of intestinal parasitic infections among food handlers in Ethiopia. All the eighteen articles were included in the analysis. This study revealed that the pooled prevalence of intestinal parasitic infections was $28.5 \%$ (95\% Cl: 27.4, 29.7). E. hystolitica /E. dispar complex 6.38 ( $95 \%$ Cl: 5.73, 7.04), A.lumbricodes 4.12 ( $95 \%$ Cl: 3.56, 4.67), and G. lamblia 3.12(95\% Cl: 2.65, 3.60) were the most common intestinal parasitic infections in this study. Untrimmed fingernail 3.04 (95\% Cl: 2.19, 4.22), do not washing hands after defecation 2.71 (95\% Cl: 1.93, 3.82), do not washing hands after touching any body parts 2.41 (95\% Cl: 1.64, 3.56), do not made medical checkup 2.26 (95\% Cl: 1.57, 3.25), and do not receive food safety training 1.79 (95\% Cl: $1.30,2.45)$ were factors significantly and positively associated with intestinal parasitic infections.

Conclusion: Parasitic infections among food handlers were significantly high. Untrimmed fingernail, do not washing hands after defecation, do not washing hands after touching any body parts, do not made regular medical checkup and do not receive food safety training were factors that increase the prevalence of intestinal parasitic infections.
\end{abstract}

Keywords: Prevalence, Intestinal parasite, Associated factors, Meta-analysis, Systematic review, Ethiopia

\footnotetext{
*Correspondence: asmamawdemis@gmail.com

${ }^{4}$ Department of Nursing, College of Health Sciences, Woldia University,

P.O.Box:400, Woldia, Ethiopia

Full list of author information is available at the end of the article
}

(c) The Author(s). 2020 Open Access This article is distributed under the terms of the Creative Commons Attribution 4.0 International License (http://creativecommons.org/licenses/by/4.0/), which permits unrestricted use, distribution, and reproduction in any medium, provided you give appropriate credit to the original author(s) and the source, provide a link to the Creative Commons license, and indicate if changes were made. The Creative Commons Public Domain Dedication waiver (http://creativecommons.org/publicdomain/zero/1.0/) applies to the data made available in this article, unless otherwise stated. 


\section{Background}

Food-borne infections are common public health problems, which become a significant public health issue all over the world [1]. The related problems are high in the low and middle-income countries, due to the difficulties in adopting optimal hygienic practices during food handling [2].

Intestinal parasites in Ethiopia are widespread and the loss of human life and suffering is enormous just like other low and middle-income countries. Several foodborne disease outbreaks are associated with the poor personal hygiene of people handling foodstuffs [3]. Lack of clean and safe water, high population density, lack of proper disposal of waste, noncompliance with health standards (social and individual), lack of adequate washing of vegetables, and lack of well-cooked meat lead to a high prevalence of intestinal parasites [4-6].

A lot of communicable diseases and microorganisms can enter the body through foods and cause infection. Intestinal parasites are one of the common agents to cause intestinal infection among food handlers [7]. The dominant intestinal protozoa and helminths parasites in Ethiopia are Giardia lamblia (G.lamblia), Entamoebahistolytica/ dispar (E.histolytica/dispar), Ascarislumbricoides (A.lumbricoides) and Trichuristrichuria (T.trichuria) [8].

Studies in Ethiopia showed that the prevalence of intestinal parasitic infection among food handlers working at University student cafeteria's and public food establishment area such as Haramaya University cafeterias (14.3\%) [9], East and West Gojjam prison (61.9\%) [10], Wollo University student's cafeteria (15\%) [11], Aksum Town (14.5\%) [12], Jimma University Specialized Hospital (33\%) [13], Addis Ababa University Students' Cafeteria (45.3\%) [7], Hawassa University (20.6\%) [14], and Mekelle University student's cafeteria (52.4\%) [2]. However, the prevalence reflected in these small and fragmented studies varied widely and remained inconclusive. Besides prevalence, identifying modifiable risk factors is a critical step in identifying potential interventions. The lack of a nationwide study that determines the prevalence and factors associated with intestinal parasitic infections among food handlers working in higher public university student's cafeterias and public food establishments is a significant gap.

Therefore, this systematic review and meta-analysis aimed to determine the pooled prevalence of intestinal parasite and associated factors among food handlers working in higher public university student cafeterias and public food establishments' using available studies in Ethiopia. The findings from this systematic review will highlight the prevalence and factors associated with intestinal parasitic infections among food handlers working in higher public university student's cafeterias and public food establishments' in Ethiopia.

\section{Methods}

\section{Study design and search strategies}

This systematic review and meta-analysis was conducted to compute the pooled prevalence and factors associated with intestinal parasitic infections among food handlers working in higher public University student's cafeterias and public food establishments in Ethiopia. Ethiopia is located in the eastern part of Africa with estimated population of $106,059,710$ with $20.2 \%$ living in the urban area $[15,16]$. The Preferred Reporting Items for Systematic Reviews and Meta-Analysis (PRISMA) checklist was used to present and report the result of the study [17]. We searched articles written in English on international databases: PubMed/ Medline, Science Direct, Web of Science Google Scholar, Hinari, and Cochrane Library [18]. Besides, Gray literature was searched through the review of available references. Besides, unpublished papers in the field of our systematic review and meta-analysis were included, online repository library including Addis Ababa, Mekelle University, Jimma University, Haramaya University, Hawassa University, and University of Gondar digital library was searched. We searched the included literature within the time interval of March 1-May1, 2019. The core search terms and phrases were "prevalence", "intestinal parasite", "associated factors", "food handlers", "University student cafeterias", public food establishment's" and "Ethiopia". The search terms were used separately and in combination using Boolean operators like "OR" or "AND”.

\section{Inclusion and exclusion criteria Inclusion criteria}

Studies published until May 1, 2019.

All observational study designs (i.e., cross-sectional, case-control and cohort) reporting the prevalence of IPI among food handlers.

Both published and unpublished articles reported in the English language.

Only studies involving among food handler working at university student cafeteria's and public food establishments in Ethiopia.

\section{Exclusion criteria}

Articles which were not fully accessed (full texts not available, no responding of contacting of the corresponding author via email two times).

\section{Outcomes of the study}

The measurement outcome of this study has two main outcome variables. Intestinal parasitic infection is the primary outcome of the study whereas associated factors of IPs among food handlers working at university student cafeterias was the second outcome variable. Intestinal parasitic infection is defined as infection caused by 
one or more parasite [19]. The prevalence of IPIs was computed as the total number of IPIs cases divided by the total number of food handlers involving in the study multiplied by 100 . The association between IPIs and associated factors were calculated in the form of the log odds ratio. The odds ratio was calculated for the common associated factors of the reported studies. The most common associated factors included in this systematic review and meta-analysis were fingernail trimming, hand washing after defecation, hand washing after touching any body parts, regular medical checkup, and food safety training.

\section{Data extraction}

Data extraction was implemented using a standard and extraction format adapted from the JBI data extraction format. Two authors (BA and AD) were involved independently to extract all the necessary information's from both published and unpublished studies and recorded by Microsoft excel spreadsheet. During the time of data extraction, discrepancies between two authors were resolved through discussion and consensus. This systematic review and meta-analysis included two primary outcomes. During data collection, the first outcome variable included the prevalence of IPs with 95\% CI, response rate, sample size, study design, and study area. Finally, data was extracted via a Microsoft Excel spreadsheet and the log odds ratio of each associated factors were calculated.

\section{Quality assessment}

The adopted Newcastle-Ottawa Scale (NOS) quality assesment tool was used for cross-sectional, cohort and case-control studies to assess the quality of each study. In this systematic review and meta-analysis, all the included articles were cross-sectional studies. Methodological quality, comparability and the outcome and statistical analysis of the study were the three major assessment tools that we used to declare the quality of the study. Moreover, studies scored a scale of $\geq 6$ out of 10 was considered as having good quality. Two authors (BA and $\mathrm{AD}$ ) independently assessed the quality of each original study using the adopted Newcastle-Ottawa Scale (NOS) quality assessment tool. During quality appraisal of the articles, any discrepancies between the two authors were handled and resolved by taking the second group authors (GG and GD). All of the studies were included based on the adopted NewcastleOttawa Scale (NOS) quality assessment criteria.

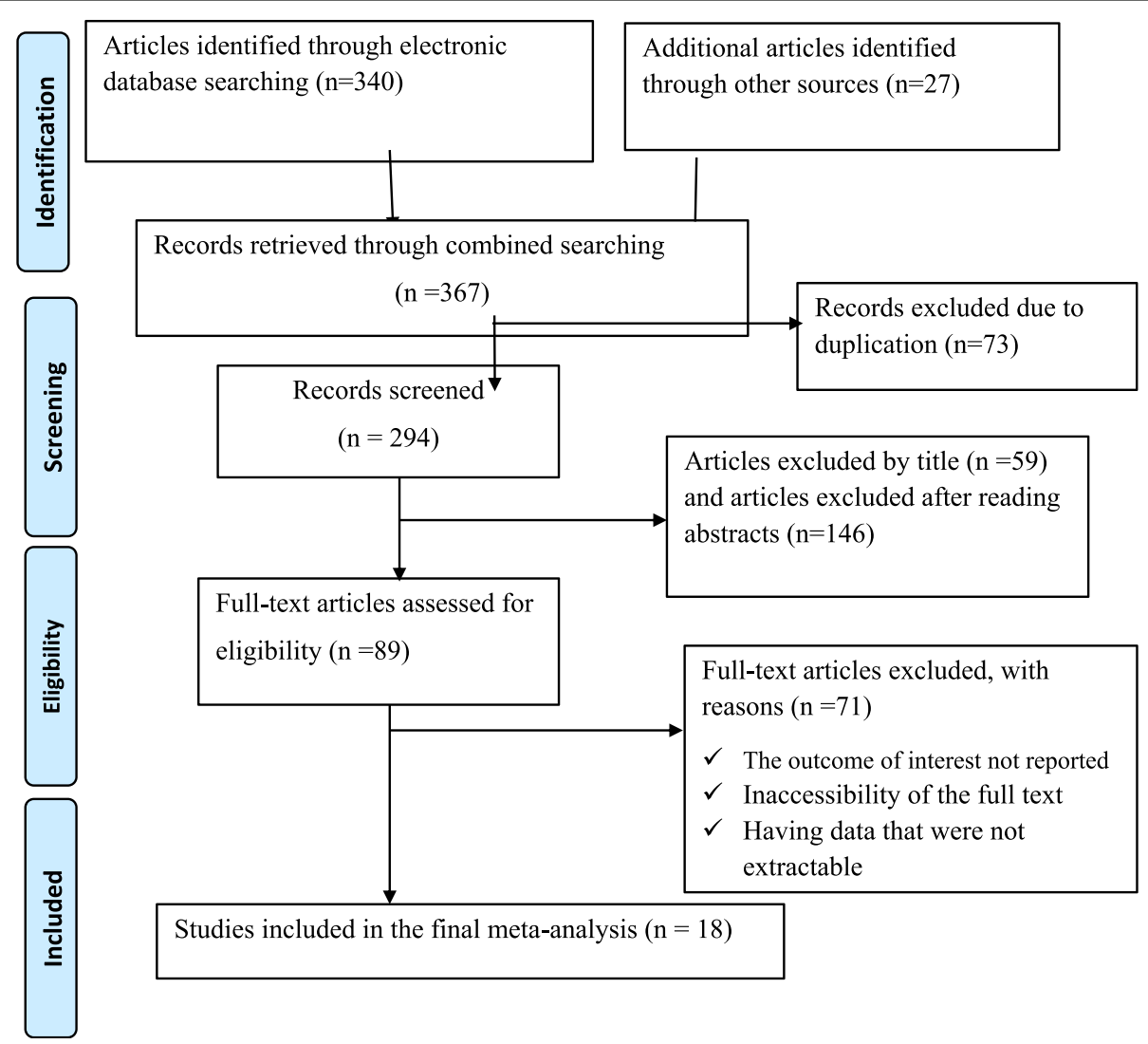

Fig. 1 Flow chart of study selection for systematic review and meta-analysis prevalence and factors associated with intestinal parasitic infections among food handlers working in higher public university students cafeterias in Ethiopia 


\section{Data analysis}

Random effect model was applied to estimate the pooled prevalence of IPs. After extraction of the articles in Microsoft Excel spreadsheet format, the analysis was carried out using STATA version 14 statistical software. Cochrane Q-test and $I^{2}$ statistics were computed to assess heterogeneity among studies [20]. After computing the statistics, results showed there is significant heterogeneity among studies $\left(I^{2}=96.80 \%, p<0.001\right)$. To compute the overall proportion of IPs, through back-transform of the weighted mean of the transformed proportions arcsine variance weights and Dersimonian-Laird weights for fixed-effects model and random effect model respectively $[21,22]$. Subgroup analysis was done based on the study setting (University vs. public food establishments) and sample size to minimize the random variations between the point estimates of the primary study. Forest plot format was used to present the pooled point prevalence with $95 \% \mathrm{Cl}$. For associations, a log odds ratio was used to decide the association between associated factors and IPIs among food handlers in the included studies.

\section{Results}

The electronic online search and other sources yielded 367 records regarding the prevalence and factors associated with intestinal parasitic infections among food handlers in Ethiopia, of which 73 duplicate records were identified and removed. Title and abstract screening resulted in the exclusion of 205 irrelevant articles. Therefore, 89 full-text articles were accessed and assessed for eligibility based on the preset criteria, which resulted in the further exclusion of 71 articles primarily due to the outcome of interest not reported, inaccessibility of the full text and having data that were not extractable. Finally, a total of 18 studies meet the eligibility criteria and were included in the meta-analysis. This systematic review and meta-analysis consist of eighteen cross sectional studies (Fig. 1).

\section{Characteristics of original studies}

Among 18 studies which were published in Ethiopia from 2000 to 2019, 5049 study participants were involved to determine the pooled prevalence of IPIs among food handlers. Regarding the study design, almost all the studies are cross-sectional. The sample size of the studies ranged from 94 to 417. The lowest prevalence of IPIs among food handlers were reported in studies conducted in Wollo University student's cafeteria (15\%) [11], Awi Amhara (14.75\%) [23], and Aksum town, Tigray (14\%) [12], whereas the highest prevalence $(61.78 \%)$ was reported in a study conducted at East and West Gojjam public prison [10]. Seven of the studies were from Amhara region [10, 11, 23-27], five from SNNP region [8, 14, 28-30], three from Oromia region [9, 13], one from Addis Ababa (7) and two from Tigray region $[2,12]$. However, there were no studies reported from Benishangul Gumuz, Harari and Gambela regions, and Dire Dawa. Regarding quality score, the quality score of each original study ranged from a low of five to a high of eight (Table 1).

Table 1 Characteristics of studies included in the systematic review and meta-analysis

\begin{tabular}{lllllll}
\hline Author & Publication Year & Region & Study Area & Sample Size & Quality score & Prevalence with 95\% \\
\hline Solomon et al., [8] & 2018 & SNNP & Wolaitasodo town & 387 & 6 & $40(35,45)$ \\
Maram et al., [9] & 2018 & Oromia & Haramaya University & 417 & 6 & $25.2(21,29)$ \\
Asires et al., [10] & 2019 & Amhara & Debre Markos Prison & 416 & 6 & $62(57,66)$ \\
Kebede et al., [11] & 2019 & Amhara & Wollo University & 200 & 7 & $15(10,20)$ \\
Gezehegn et al., [12] & 2017 & Tigray & Aksum Town & 400 & 7 & $15(11,18)$ \\
Mama et al., [31] & 2016 & SNNP & Arba Minch University & 378 & 6 & $32(28,37)$ \\
Tefera et al., [28] & 2014 & Oromia & Yebu Town & 118 & 5 & $44(35,53)$ \\
Andargie et al., [25] & 2008 & Amhara & Gondar Town & 127 & 7 & $29(21,37)$ \\
Girma et al., [13] & 2017 & Oromia & JimmaUniversity & 94 & 6 & $33(23,42)$ \\
Aklilu et al., [7] & 2014 & Addis Ababa & Addis Ababa University & 172 & 7 & $45(37,53)$ \\
Abera et al., [24] & 2010 & Amhara & Bahir Dar Town & 384 & 8 & $41(36,46)$ \\
Gebreyesus et al., [2] & 2014 & Tigray & Mekelle University & 307 & 6 & $52(47,58)$ \\
Dagnew et al., [26] & 2012 & Amhara & University of Gondar & 200 & 7 & $25(19,31)$ \\
Desta et al., [14] & 2014 & SNNP & Hawassa University & 272 & 5 & $21(16,25)$ \\
Wadilo et al., [32] & 2016 & SNNP & Wolaitasodo town & 288 & 5 & $34(28,39)$ \\
Alemu et al. [23] & 2019 & Amhara & Chagni town & 400 & 6 & $15(11,18)$ \\
Kumma et al. [30] & 2019 & SNNP & Wolaitasodo university & 233 & 5 & 7 \\
Demis et al. [27] & 2019 & Amhara & Woldia university & 256 & $7(18,29)$ & $17(12,21)$ \\
\hline
\end{tabular}




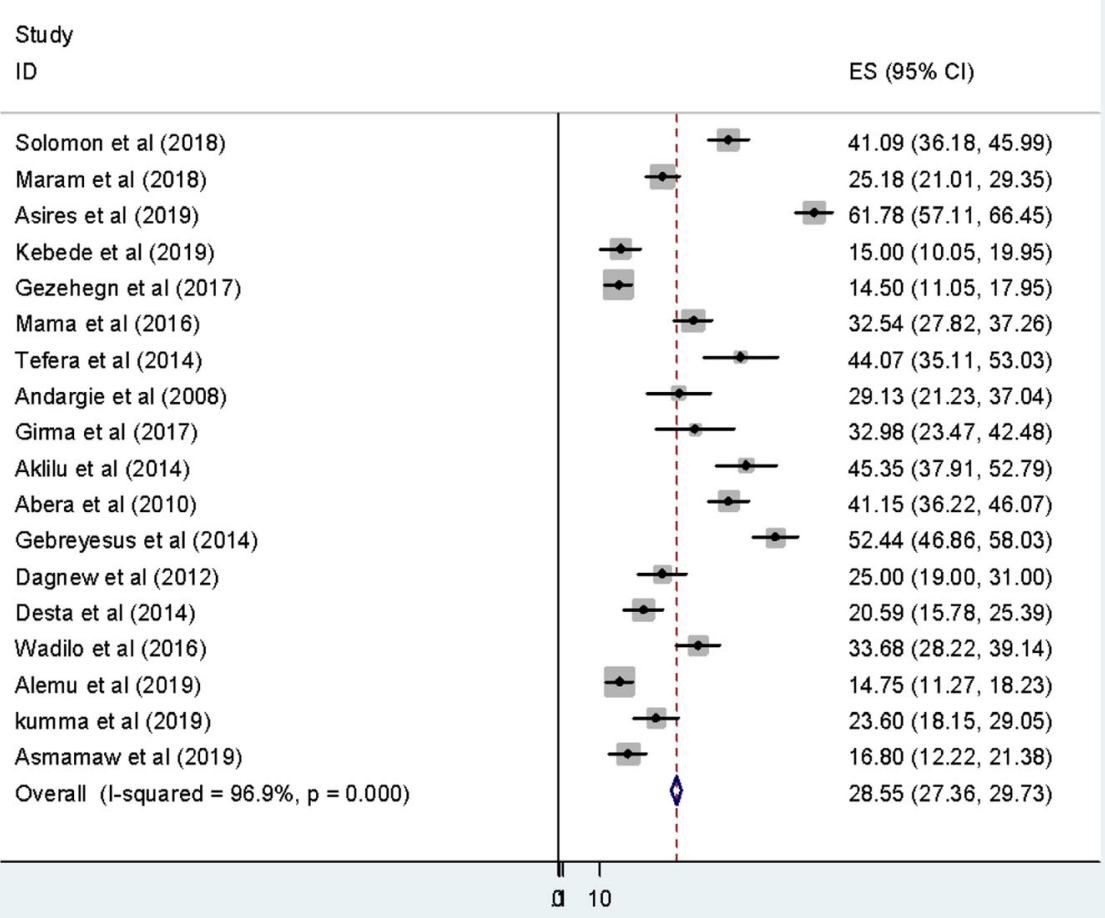

Fig. 2 Forest plot of the pooled prevalence intestinal parasitic infections among food handlers in Ethiopia

Prevalence intestinal parasitic infections among food handlers working in higher public university student's cafeterias and public food establishments in Ethiopia

The eighteen included studies revealed that prevalence intestinal parasitic infections among food handlers working in higher public University students cafeterias and public food establishments were 28.5\% (95\% CI, 27.4, 29.7) (Fig. 2). High heterogeneity was observed across the included studies $\left(\mathrm{I}^{2}=96.90, P<0.001\right)$. As a result, a random-effects model was employed to estimate the pooled prevalence of intestinal parasitic infection among food handlers in Ethiopia.

\section{Heterogeneity and publication bias}

The existence of heterogeneity and publication bias was determined within the included studies. Consequently, there was considerable heterogeneity across fifteen included studies in this meta-analysis $\left(\mathrm{I}^{2}=96.9 \%\right)$. Publication bias was assessed using Begg's and Egger's tests, showing no statistically significant for estimating the

Table 2 Subgroup pooled prevalence of intestinal parasite among food handlers in Ethiopia, 2019( $n=18)$

\begin{tabular}{|c|c|c|c|c|}
\hline Variables & Characteristics & Included studies & Sample size & Prevalence with $(95 \% \mathrm{Cl})$ \\
\hline \multirow[t]{4}{*}{ Region } & Amhara & 7 & 1983 & $27.55(25.73,29.37)$ \\
\hline & Oromia & 3 & 629 & $29.14(25.63,32.65)$ \\
\hline & Addis Ababa and Tigray & 3 & 879 & $27.72(24.99,30.45)$ \\
\hline & SNNP & 5 & 1558 & $30.39(28.13,32.64)$ \\
\hline \multirow[t]{2}{*}{ Sample size } & $>200$ & 12 & 4138 & $28.71(27.41,30.01)$ \\
\hline & $\leq 200$ & 6 & 911 & $27.78(24.97,30.60)$ \\
\hline \multirow[t]{2}{*}{ Study site } & University cafeterias & 10 & 2529 & $26.81(25.14,28.48)$ \\
\hline & Public town food establishments & 8 & 2520 & $30.28(28.61,31.95)$ \\
\hline Overall & & 18 & 5,049 & $28.55(27.36,29.73)$ \\
\hline
\end{tabular}


Table 3 Pooled prevalence of some common intestinal parasites among food handlers in Ethiopia

\begin{tabular}{lll}
\hline Types of intestinal parasites & Pooled prevalence $(95 \% \mathrm{Cl})$ & I-Squared \\
\hline A.lumbricoides & $4.12(3.56,4.67)$ & $95.3 \%, p<0.001$ \\
E. hystolitica /E. dispar complex & $6.38(5.73,7.04)$ & $95.0 \%, p<0.001$ \\
G. lamblia & $3.12(2.65,3.60)$ & $76.8 \%, p<0.001$ \\
Taenia spp. & $1.07(0.75,1.40)$ & $73.9 \%, p<0.001$ \\
Hookworms & $1.70(1.31,2.09)$ & $83.1 \%, p<0.001$ \\
T. trichuria & $0.84(0.42,1.26)$ & $12.7 \%, p=0.33$ \\
H. nana & $1.03(0.66,1.41)$ & $90.7 \%, p<0.001$ \\
E. vermicularis & $2.69(1.43,3.96)$ & $53.0 \%, p=0.119$ \\
S. mansoni & $0.70(0.34,1.07)$ & $51.1 \%, p=0.056$ \\
\hline
\end{tabular}

prevalence of IPIs among food handlers $(p=0.081)$ and $(P=0.075)$ respectively.

\section{Subgroup analysis}

We performed a subgroup analysis by taking different factors. The region of the country, sample size, study area (University versus Town) was factored we considered to perform subgroup analysis. Consequently, the subgroup analysis of this study indicated that the highest prevalence of IPIs was observed in Amhara region, $27.55 \%$ (95\% CI: 25.73, 29.37), SNNP, 30.39\% (95\% CI: 28.13, 32.64), and Oromia, 29.14\% (95\% Cl: 25.63, 32.65) respectively, whereas the lowest prevalence was observed in Tigray and Addis Ababa with the prevalence

\section{Study}

ID

$\mathrm{ES}(95 \% \mathrm{Cl})$

Solomon et al (2018)

Maram et al (2018)

Asires et al (2019)

Kebede et al (2019)

Mama et al (2016)

Tefera et al (2014)

Girma et al (2017)

Alemu et al (2019)

kumma et al (2019)

Asmamaw et al (2019)

Overall $($ I-squared $=49.5 \%, p=0.037)$

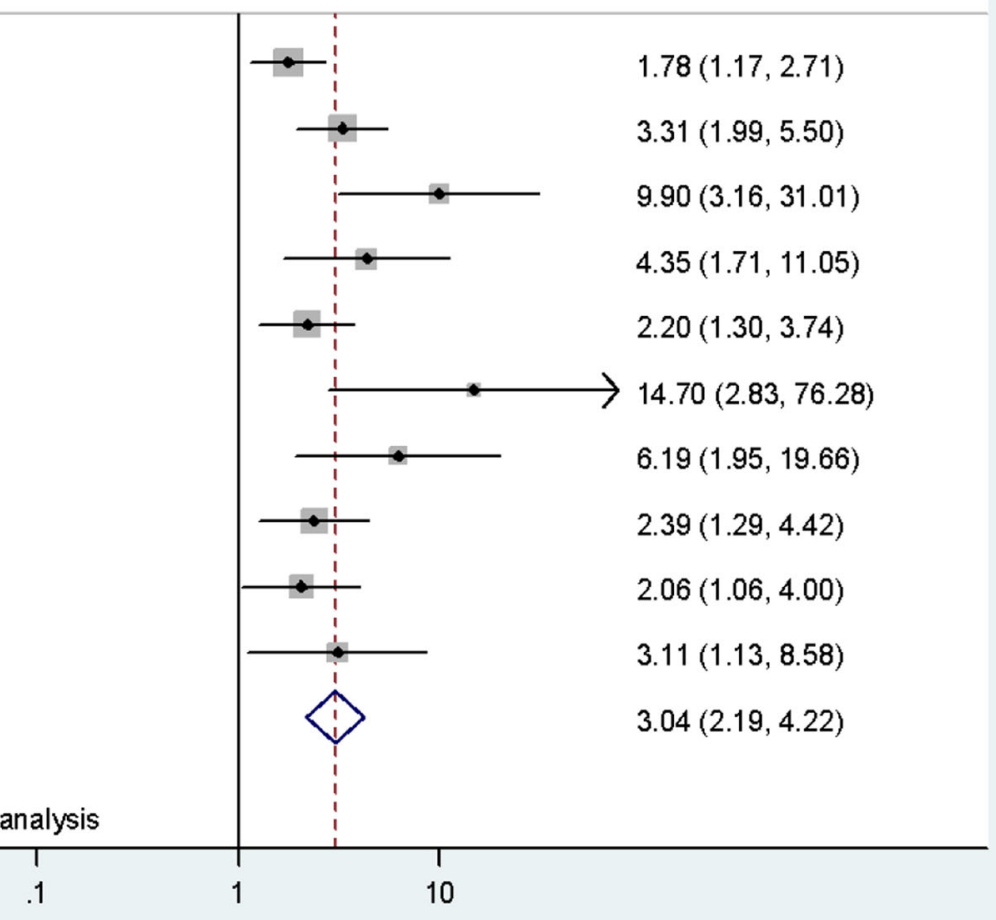

NOTE: Weights are from random effects analysis 
of $27.72 \%$ (95\% CI: $24.99,30.45)$ respectively (Table 3 ). Furthermore, subgroup analysis was performed based on the sample size of the studies. The pooled prevalence of IPIs was higher in studies having a sample size (n) > 200, 28.71\% (95\% CI: 27.41, 30.01) compared to those having a sample size $(\mathrm{n}) \leq 2$ 00, 27.78\% (95\% CI: 24.97, 30.60). Moreover, subgroup analysis was performed based on study area/site where food handlers working in university versus public food establishments, resulting in a pooled prevalence of intestinal parasite $26.81 \%$ (95\% CI: $25.14,28.48)$ and $30.28 \%$ (95\% CI: 28.61, 31.95), respectively (Table 2).

Common intestinal parasites among food handlers working in higher public university student's cafeterias and public food establishments in Ethiopia

Furthermore, in this meta-analysis, the overall pooled prevalence of the common type of intestinal parasites among food handlers was observed from 18 studies as showed (Table S1). The pooled prevalence of E. hystolitica/E. dispar complex 6.38\%(95\% CI:5.73, 7.04), A. lumbricoides 4.12\%(95\% CI:3.56,
4.67), G. lamblia 3.12\%(95\% CI:2.65, 3.60), E.vermicularis 2.69\%(95\% CI:1.43,3.96), Hookworm 1.70\%(95\% CI:1.31, 2.09), Taenia species 1.07\% (95\% CI:0.75, 1.40), H.nana 1.03\% (95\% CI:0.66, 1.41), T. trichuria 0.84\% (95\% CI:0.42, 1.26), and S. mansoni 0.70\%(95\% CI:0.34, 1.07) was found from food handlers in Ethiopia (Table 3).

\section{Factors associated with intestinal parasitic infections among food handlers in Ethiopia}

We observed the association between fingernail trimming and intestinal parasitic infections in this metaanalysis [8-11, 13, 23, 27-30]. These ten studies finding showed that the occurrence of intestinal parasitic infections was significantly associated with nail trimming habits of food handlers. Hence, the odds of intestinal parasitic infections occurrence was 3.04 times higher among food handlers who hadn't regular nail trimming habits as compared to those who had regular nail trimming habits (OR: 3.04 95\% CI: 2.19, 4.22). The finding of these test statistics revealed that there is low heterogeneity $\left(\mathrm{I}^{2}=49.5 \%\right.$ and $\left.P=0.037\right)$. As a result, a random effect

Study

ID

$\mathrm{ES}(95 \% \mathrm{Cl})$

Solomon et al (2018)

Maram et al (2018)

Asires et al (2019)

Kebede et al (2019)

Mama et al (2016)

Tefera et al (2014)

Girma et al (2017)

Alemu et al (2019)

Asmamaw et al (2019)

Overall (l-squared $=85.2 \%, p=0.000)$

NOTE: Weights are from random effects analysis

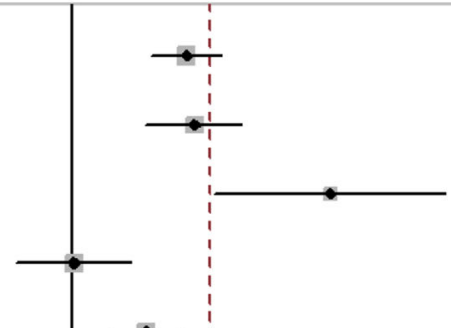

$2.30(1.78,2.97)$

$2.43(1.72,3.44)$

$6.55(2.83,15.16)$

$1.01(0.67,1.52)$

$1.71(1.34,2.18)$

$2.20(1.31,3.69)$

$9.00(5.04,16.07)$

$3.39(2.27,5.07)$

$3.24(2.04,5.15)$

$2.71(1.93,3.82)$

NoTE: Weights are from random effect

10

Fig. 4 The pooled odds ratio between hands washing after defecation with intestinal parasitic infection among food handlers in Ethiopia 
model was implemented to determine the association. Possibility of publication bias was detected using Begg's and Egger's tests with a $p$-value of 0.020 and 0.002 respectively (Fig. 3).

The association between handwashing after defecation with intestinal parasitic infections was evaluated by using seven studies $[8,10,11,13,23,25,27-29]$. This metaanalysis result revealed that hand washing after defecation is not significantly associated with intestinal parasitic infection [11]. However, the odds of having intestinal parasitic infections was 2.71 higher among food handlers who hadn't hand washing after defecation as compared with the counterparts (OR: 2.71, 95\%; CI: 1.93, 3.82) (Fig. 4). This studies showed that there was the existence of high heterogeneity $\left(\mathrm{I}^{2}=85.2 \%\right.$ and $\left.P<0.001\right)$ therefore, random-effect metaanalysis was considered. No publication bias was detected using Begg's and Egger's tests with a $p$-value of 0.118 and 0.107 respectively.

The association between handwashing after touching any body parts with intestinal parasitic infection among food handlers was computed by using eight studies $[8,9$,
$12,13,27-30]$. The overall finding of this study showed that food handlers who didn't wash their hands after touching any body parts were 2.41 higher than their counterparts (OR:2.41, 95\% CI:1.64, 3.55) (Fig. 5). Moderate heterogeneity $\left(\mathrm{I}^{2}=67.1 \%\right.$; $P$-value $\left.=0.003\right)$ was observed among the included studies; hence, a random effect metaanalysis model was employed. Moreover, publication bias was detected using the Begg's and Egger's tests with a $p$ value of 0.266 and 0.376 respectively.

Similarly, the association between regular medical checkup and intestinal parasitic infection among food handlers in Ethiopia were computed in this metaanalysis [9, 11-13, 23, 27]. The overall meta-analysis report showed that food handlers who hadn't regular medical checkup were 2.67 more likely to have intestinal parasitic infections than those who had regular medical checkup (OR: 2.67, 95\% CI: 1.51, 4.71) (Fig. 6). Low heterogeneity $\left(\mathrm{I}^{2}=51.2 \% ; p\right.$-value $\left.=0.069\right)$ was detected among the included studies; for this reason, a random effect meta-analysis model was computed. Furthermore, no possible publication bias was detected using the

Study

ID

$\mathrm{ES}(95 \% \mathrm{Cl})$

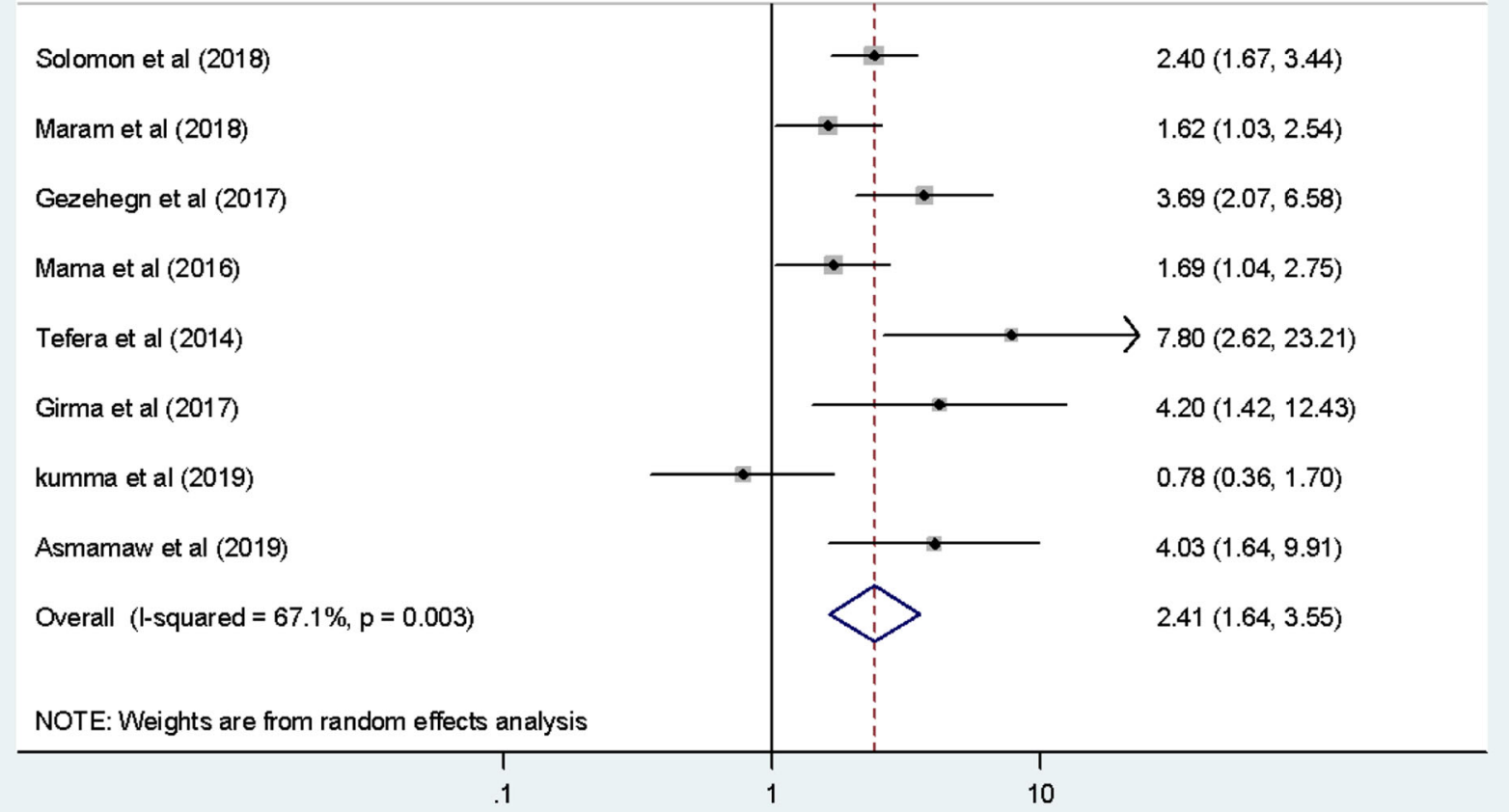

Fig. 5 The pooled odds ratio of the association between handwashing after touching any body parts with intestinal parasitic infection among food handlers in Ethiopia 


\section{Study}

ID

$\mathrm{ES}\{95 \% \mathrm{Cl}\}$

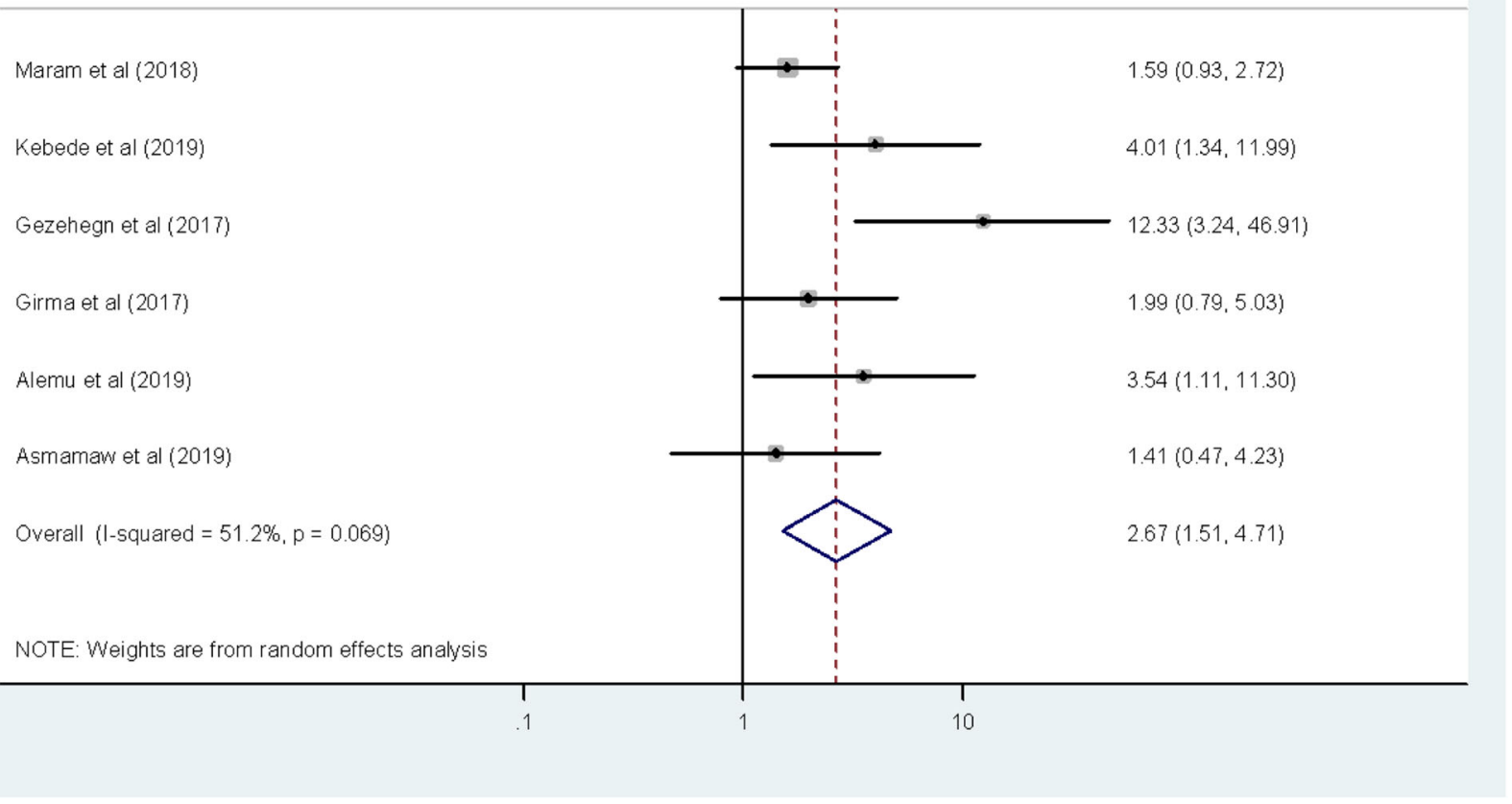

Fig. 6 The pooled odds ratio of the association between regular medical checkup and intestinal parasitic infections among food handlers in Ethiopia

Begg's and Egger's tests with a $p$-value of 0.133 and 0.103 respectively.

Lastly, we employed the association between food safety training and intestinal parasitic infections. We included five studies that examined the association between intestinal parasitic infections with food safety training among food handlers $[8,9,11,13,27]$. The pooled result of this meta-analysis indicated that food handlers who didn't receive food safety training were 2.11 more likely to have intestinal parasitic infections as compared with those who received food safety training (OR: 2.11, 95\%CI: 1.18, 3.77) (Fig. 7). In this metaanalysis, the included studies were characterized by low heterogeneity $\left(\mathrm{I}^{2}=63.6 \% ; P=0.027\right)$. Furthermore, low publication bias was detected using the Begg's and Egger's tests with a $p$-value of 1 and 0.248 respectively.

\section{Discussion}

Intestinal parasitic infection is one of the most common causes of morbidity and mortality among food handlers in Ethiopia [33]. Ballpark figuring of the pooled prevalence of intestinal parasitic infection and its associated factors in Ethiopia may give attention to policymakers to take a corrective action based on the evidence. Hence, this systematic review and meta-analysis were conducted to estimate the overall pooled prevalence of IPIs and its associated factors among food handlers in Ethiopia. The overall pooled prevalence of IPIs obtained from this meta-analysis showed that (28.5\%; 95\% CI: 27.4, 29.7) among food handlers in Ethiopia were suffered from IPIs. The finding of this metaanalysis was higher than the study conducted in Iran 8.8\% Southwest [34], 15.5\% in Sari, Northern [35], and 10.4\% Shiraz [36]. On the other hand, this study finding showed that the result is in line with the study done Northwest Ethiopia (27.7\%) IPIs from clinically suspected patients [37]. The possible justification for the above disparity could be credited to methodological variation in the assessment of prevalence. The discrepancy in the prevalence of IPIs this review and meta-analysis study and other African countries could be rationalized by the dissimilarity in socio-demographics, personal and environmental hygiene practice.

The subgroup analysis of this study showed that the highest prevalence of Intestinal parasitic infection was 


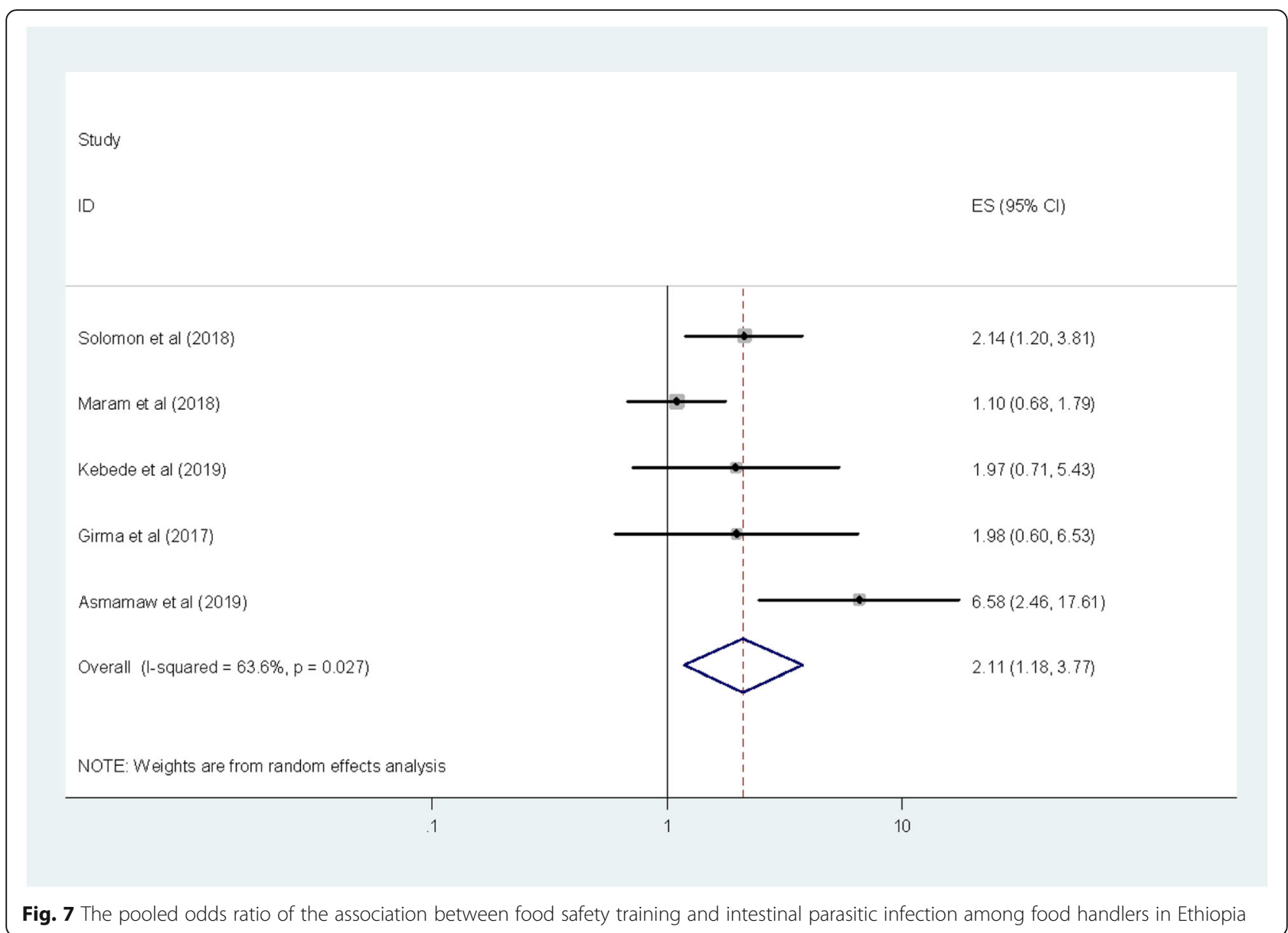

observed in SNNP, 30.39\% (95\% CI: 28.13, 32.64), Oromia, 29.14\% (95\% Cl: 25.63, 32.65) and Amhara region, 27.55\% (95\% CI: 25.73, 29.37), whereas the lowest prevalence was observed in Tigray and Addis Ababa with the prevalence of $27.72 \%$ (95\% CI: $24.99,30.45)$ respectively. The possible explanation for this variations across the region might be due to sociodemographic, environmental, behavioural characteristics of food handlers and quality of food establishments.

In this meta-analysis, the pooled prevalence of intestinal protozoa infection was E.histolytica 6.38\% (5.73, 7.04), and G. Lamblia $3.12 \%(2.65,3.60)$. The finding of this meta-analysis in line with the study done in Iran $G$. lamblia (4.52\%), and E. hystolitica /E. dispar complex (1.39\%) [34]. However, our study finding was lower than the study done in Libya and sari Northern Iran, E. hystolitica /E. dispar complex 19.9\% G. lamblia 4.6\% [38], and in Giardia lamblia (1.6\%) respectively [35].

In this meta-analysis Taenia species $1.07 \%(0.75,1.40)$, Hookworm 1.70\% (1.31, 2.09), T. trichuria 0.84\% (0.42, 1.26), H.nana $1.09 \%(0.66,1.41)$, E. vermicularis $2.69 \%$ $(1.43,3.96)$, and $S$. mansoni $0.70 \%(0.34,1.07)$ were the commonest protozoan infections. The finding of this study was relatively consistency with studies in Southwest Iran, H.nana (1.29\%), A.lumbricoides (0.57\%), and E.vermicularis, T. trichiura, S. stercoralis was each less than $0.5 \%$ [34]. However, a study conducted in Sari, Northern Iran showed that $H$. nana (1.9\%) was the only helminthic infection [35]. Furthermore, a study from clinically suspected patients in northwest Ethiopia was hookworm 21.1\%, and A.lumbricoids 3.9\% [37]. Besides, a survey study in Ethiopia reported that $A$.lumbricoides 9.9\%, hookworm 9.7\%, and T.trichiura $2.6 \%$ were the commonest intestinal helminths [39].

Under subgroup analysis, the result of this study showed that the highest prevalence of IPIs was observed in SNNP, 30.73\% (95\% CI: 28.34, 33.11), followed by Oromia $28.81 \%$ (95\% CI: 25.69, 31.94), then Tigray and Addis Ababa 27.72\%(95\% CI: 24.99, 30.45) whereas Amhara region was the lowest prevalent region with the prevalence of $27.55 \%$ (95\% CI: 25.73, 29.37). The potential justification for this difference might be due to the distinction in socio-demographic, environmental, geographical and behavioural characteristics. 
This systematic review and meta-analysis study is aimed to identify factors associated with intestinal parasitic infection among food handlers in Ethiopia. In this study, fingernail trimming, hand washing after defecation, hand washing after touching any body parts, regular medical checkup, and food safety training were significantly associated with intestinal parasitic infections.

The odds of not having handwashing after defecation were 2.71 times more likely to develop intestinal parasitic infections than their counterparts. This finding is supported by the studies conducted in Kenya [40], Gambia [41], Pakistan [42] and Ethiopia [30, 43, 44]. This might be due to feco-oral microorganism transmissions. The odds of untrimmed fingernail were 3.04 times more likely to acquire intestinal parasitic infections than their counterparts. This finding is supported by the studies conducted in Ethiopia [30,43,44]. This might be due to the fact that untrimmed fingernail may contain dust particles and microorganisms which facilitates in easily transmissions of microorganisms feco-orally.

The odds of didn't take food safety training were 1.79 times more likely to be infected with intestinal parasites than those food handlers who took food safety training. This finding supported by the studies conducted in Bangladesh [45] and Saud Arabia [46]. This might be due to the fact those food handlers who didn't take food safety training may lack the necessary knowledge and practice towards transmission and prevention of microorganisms.

\section{Limitations of the study}

Like other meta-analyses, this study shares its own limitation. Even though the finding of published articles other than English is consistent with others but we only considered articles published only in English languages. Besides, all of the studies included in this review were cross-sectional; as a result, the outcome variable might be affected by other confounding variables.

\section{Conclusion}

In this study, intestinal parasitic infection among food handlers in Ethiopia was significantly high. Untrimmed fingernail, do not washing hands after defecation, do not washing hands after touching any body parts, do not made regular medical checkup and do not receive food safety training were factors that increase the prevalence of intestinal parasitic infections. Therefore, based on the study findings, the authors recommend particular emphasis shall be given to the periodic and regular medical care, health educations about personal hygiene, hand washing practice, and food safety are recommended for food handlers.

\section{Supplementary information}

Supplementary information accompanies this paper at https://doi.org/10. 1186/s12879-020-4884-4

Additional file 1: Table S1. some common intestinal parasite among food handlers from each individual study.

\section{Abbreviations}

Cl: Confidence Interval; IPs: Intestinal parasites; OR: Odd Ratio;

PRISMA: Preferred Reporting Items for Systematic Reviews and Meta-Analysis; SNNPR: South Nation Nationalities and Peoples Region

\section{Acknowledgments}

Not applicable

Authors' contributions

$A D$ and $B A$ developed the draft proposal under the supervision of $G G$ and GD. All authors (AD, BA, GG \& GD) critically reviewed, provided substantive feedback and contributed to the intellectual content of this paper and made substantial contributions to the conception, conceptualization and manuscript preparation of this systematic review. All authors read and approved the final manuscript.

Funding

Not applicable

Availability of data and materials

All related data has been presented within the manuscript. The dataset supporting the conclusions of this article is available from the authors on request.

Ethics approval and consent to participate

Not applicable

Consent for publication

Not applicable.

Competing interests

The authors declare that they have no competing interests.

\section{Author details}

${ }^{1}$ Department of Medical Laboratory Sciences, College of Health Sciences, Woldia University, P.O.Box:400, Woldia, Ethiopia. ${ }^{2}$ Department of Midwifery, College of Health Sciences, Woldia University, P.O.Box:400, Woldia, Ethiopia. ${ }^{3}$ Department of Public Health, College of Health Sciences, Woldia University, P.O.Box:400, Woldia, Ethiopia. ${ }^{4}$ Department of Nursing, College of Health Sciences, Woldia University, P.O.Box:400, Woldia, Ethiopia.

Received: 23 July 2019 Accepted: 12 February 2020

Published online: 19 February 2020

References

1. Eshetu L, Dabsu R, Tadele G. Prevalence of intestinal parasites and its risk factors among food handlers in food services in Nekemte town, West Oromia, Ethiopia. Res Rep Trop Med. 2019;10:25-30 PubMed PMID: 31191082. Pubmed Central PMCID: PMC6512780.

2. Gebreyesus A, Adane K, Negash L, Asmelash T, Belay S, Alemu M, et al. Prevalence of Salmonella typhi and intestinal parasites among food handlers in Mekelle University student cafeteria, Mekelle, Ethiopia. Food Control. 2014:44:45-8.

3. Kebede Deribe KM, Gebre T, Hailu A, Ali A, Aseffa A, Davey G. The burden of neglected tropical diseases in Ethiopia, and opportunities for integrated control and elimination; 2014.

4. Mendedo EK, Berhane Y, Haile BT. Factors associated with sanitary conditions of food and drinking establishments in Addis Ababa, Ethiopia: cross-sectional study. Pan Afr Med J. 2017;28:237 PubMed PMID: 29881482 Pubmed Central PMCID: PMC5989266.

5. Adane M, Teka B, Gismu Y, Halefom G, Ademe M. Food hygiene and safety measures among food handlers in street food shops and food establishments of Dessie town, Ethiopia: A community-based cross-sectional 
study. PLoS One. 2018;13(5):e0196919 PubMed PMID: 29723288. Pubmed Central PMCID: PMC5933796.

6. Mulugeta K. Food safety and foodborne disease in 21st century homes. Canada J Infect Dis. 2003;14(5):277-80.

7. Aklilu A, Kahase D, Dessalegn M, Tarekegn N, Gebremichael S, Zenebe S, et al. Prevalence of intestinal parasites, salmonella and shigella among apparently health food handlers of Addis Ababa University student's cafeteria, Addis Ababa, Ethiopia. BMC Res Notes. 2015;8:17 PubMed PMID: 25616876. Pubmed Central PMCID: PMC4310029.

8. Solomon FB, Wada FW, Anjulo AA, Koyra HC, Tufa EG. Burden of intestinal pathogens and associated factors among asymptomatic food handlers in South Ethiopia: emphasis on salmonellosis. BMC Res Notes. 2018;11(1):502 PubMed PMID: 30041687 . Pubmed Central PMCID: PMC6056936.

9. Marami D, Hailu K, Tolera M. Prevalence and associated factors of intestinal parasitic infections among asymptomatic food handlers working at Haramaya University cafeterias, eastern Ethiopia. Ann Occup Environ Med. 2018;30:53 PubMed PMID: 30167309. Pubmed Central PMCID: PMC6103851.

10. Asires A, Wubie M, Reta A. Prevalence and associated factors of intestinal parasitic infections among food handlers at prison, east and west Gojjam, Ethiopia. Adv Med. 2019;2019:2101089 PubMed PMID: 30723752. Pubmed Central PMCID: PMC6339768.

11. Kebede E, Seid A, Akele S. Prevalence and associated risk factors of intestinal parasitic infections among asymptomatic food handlers in Wollo University student's cafeteria, Northeastern Ethiopia. BMC Res Notes. 2019;12(1):139 PubMed PMID: 30871610. Pubmed Central PMCID: PMC6419491.

12. Gezehegn D, Abay M, Tetemke D, Zelalem H, Teklay H, Baraki Z, et al. Prevalence and factors associated with intestinal parasites among food handlers of food and drinking establishments in Aksum town, Northern Ethiopia. BMC Public Health. 2017;17(1):819 PubMed PMID: 29041926. Pubmed Central PMCID: PMC5645889.

13. Girma H, Beyene G, Mekonnen Z. Prevalence of intestinal parasites among food handlers at cafeteria of Jimma University specialized hospital, Southwest Ethiopia. Asian Pac J Trop Dis. 2017;7(8):467-71.

14. Moges Desta. Prevalence of Salmonella and Shigella among food handlers in catering establishments in Hawassa University, Hawassa, Ethiopia. 2010.

15. WIKIPEDIA Geography of Ethiopia available at https://en.wikipedia.org/wiki/ Geography_of_Ethiopia. Accessed 23 Aug 2019.

16. Ayode D, McBride CM, de Heer H, Watanabe E, Gebreyesus T, Tadele G, et al. The association of beliefs about heredity with preventive and interpersonal behaviors in communities affected by podoconiosis in rural Ethiopia. Am J Trop Med Hyg. 2012;87(4):623-30 PubMed PMID: 22826482. Pubmed Central PMCID: PMC3516310.

17. Liberati A, Altman DG, Tetzlaff J, Mulrow C, Gotzsche PC, loannidis JP, et al The PRISMA statement for reporting systematic reviews and meta-analyses of studies that evaluate health care interventions: explanation and elaboration. J Clin Epidemiol. 2009;62(10):e1-34 PubMed PMID: 19631507. Epub 2009/07/28. eng.

18. Moher D, Pham B, Lawson M, Klassen T. The inclusion of reports of randomised trials published in languages other than English in systematic reviews. Health Technol Assess. 2003;7(41):1-90.

19. Kiani H. Distribution and risk factors associated with intestinal parasite infections among children with gastrointestinal disorders; 2016.

20. Rücker G, Schwarzer G, Carpenter JR, Schumacher M. Undue reliance on I 2 in assessing heterogeneity may mislead. BMC Med Res Methodol. 2008;8:79.

21. Nyaga VN, Arbyn M, Aerts M. Metaprop: a Stata command to perform metaanalysis of binomial data. Arch Public Health. 2014;72(1):39.

22. Barendregt JJ, Doi SA, Lee YY, Norman RE, Vos T. Meta-analysis of prevalence. J Epidemiol Community Health. 2013;67(11):974-8.

23. Alemu AS, Baraki AG, Alemayehu M, Yenit MK. The prevalence of intestinal parasite infection and associated factors among food handlers in eating and drinking establishments in Chagni Town, Northwest Ethiopia. BMC Res Notes. 2019;12(1):302 PubMed PMID: 31138325. Pubmed Central PMCID: PMC6540465.

24. Abera B, Biadegelgen F, Bezabih B.Prevalence of Salmonella typhi and intestinal parasites among food handlers in Bahir Dar Town, Northwest Ethiopia.Ethiop. J. Health Dev. 2010;24(1):46-50.

25. Afework Kassu GA, Tiruneh M, Moges F, Huruy K. Prevalence of Bacteria and Intestinal Parasites among Food-handlers in Gondar Town, Northwest Ethiopi; 2008.

26. Mulat Dagnew MT, Moges F, Tekeste Z. Survey of nasal carriage of Staphylococcus aureus and intestinal parasites among food handlers working at Gondar University, Northwest Ethiopia; 2012.
27. Demis A, Belay Y, Alemnew B. Prevalence and factors associated with intestinal parasitic infections among food handlers working at Woldia University Student's cafeteria, Northeastern Ethiopia; 2019.

28. Tefera T, Mebrie G. Prevalence and predictors of intestinal parasites among food handlers in Yebu town, Southwest Ethiopia. PLoS One. 2014;9(10): e1 10621 PubMed PMID: 25329050. Pubmed Central PMCID: PMC4201565.

29. Mama M, Alemu G. Prevalence and factors associated with intestinal parasitic infections among food handlers of southern Ethiopia: cross sectional study. BMC Public Health. 2016;16:105 PubMed PMID: 26831331. Pubmed Central PMCID: PMC4736149.

30. Kumma WP, Meskele W, Admasie A. Prevalence of Intestinal Parasitic Infections and Associated Factors Among Food Handlers in Wolaita Sodo University Students Caterings, Wolaita Sodo, Southern Ethiopia: A CrossSectional Study. Front Public Health. 2019;7:140. https://doi.org/10.3389/ fpubh.2019.00140 English.

31. Mama M, Alemu G. Prevalence, antimicrobial susceptibility patterns and associated risk factors of Shigella and Salmonella among food handlers in Arba Minch University, South Ethiopia. BMC Infect Dis. 2016;16(1):686 PubMed PMID: 27871241. Pubmed Central PMCID: PMC5117698.

32. Wadilo F, Solomon F, Arota A, Abraham Y. Intestinal parasitic infection and associated factors among food handlers in South Ethiopia; 2016.

33. Central Statistical Agency (Ethiopiaphic). Demographic and Health Survey Key Indicators. 2016. Available at https:/dhsprogram.com/pubs/pdf/FR328/FR328. pdf. Accessed 16 Feb 2020

34. Jasem S. Prevalence of intestinal parasitic infections among food handlers in Khuzestan, Southwest of Iran: A 10-year retrospective study. Afr J Microbiol Res. 2012;6(10):2475-2480.

35. Sharif M, Daryani A, Kia E, Rezaei F, Nasiri M, Nasrolahei M. Prevalence of intestinal parasites among food handlers of sari, northern Iran. Rev Inst Med Trop Sao Paulo. 2015;57(2):139-44 PubMed PMID: 25923893. Pubmed Central PMCID: PMC4435012

36. Hossein M. Prevalence of intestinal parasites among food-handlers in shiraz, Iran; 2015.

37. Hailu T. Current Prevalence of Intestinal Parasites Emphasis on Hookworm and Schistosoma mansoni Infections among Patients at Workemeda Health Center, Northwest Ethiopia. Clin Microbiol Open Access. 2014;03(04):155. https://doi.org/10.4172/2327-5073.1000155.

38. Ghenghesh KS, Ghanghish K, BenDarif ET, Shembesh K, Franka E. Prevalence of Entamoeba histolytica, Giardia lamblia, and Cryptosporidium spp. in Libya: 2000-2015. Libyan J Med. 2016;1 1:32088 PubMed PMID: 27363524. Pubmed Central PMCID: PMC4929352.

39. King JD, Endeshaw T, Escher E, Alemtaye G, Melaku S, Gelaye W, et al. Intestinal parasite prevalence in an area of Ethiopia after implementing the SAFE strategy, enhanced outreach services, and health extension program. PLoS Negl Trop Dis. 2013;7(6):e2223 PubMed PMID: 23755308. Pubmed Central PMCID: PMC3675016.

40. Ogolla JO. Prevalence and factors associated with intestinal protozoan and helminthic infections among certified food handlers in Eldoret town, Uasin Gishu County in Kenya. Int Clin Pathol J. 2018;6(3):127-31. https://doi.org/10. 15406/icpjl.2018.06.00171.

41. Jallow Haruna $\mathrm{S}$, et al. High prevalence of intestinal parasite carriage among food handlers in the Gambia. Int J Food Sci Biotechnol. 2017;2(1):1.

42. Khan W, Noor-un-Nisa, Khan A. Prevalence and Risk Factors Associated with Intestinal Parasitic Infections among Food Handlers of Swat, Khyber Pakhtunkhwa, Pakistan. J Food Nutr Res. 2017;5(5):331-6.

43. Eshetu L, Dabsu R, Tadele G. Prevalence of intestinal parasites and its risk factors among food handlers in food services in Nekemte town, West Oromia, Ethiopia. Res Rep Trop Med. 2019;10:25-30.

44. Abate M, Tesfa M, Adere A. The sanitary conditions of food and drink establishments in Woldia town, Northeastern Ethiopia. Ethiop J Health Dev. 2018:32(3):189-196.

45. Uddin MJ, Koehlmoos TL, Ashraf A, Khan Al, Saha NC, et al. Health needs and health-care-seeking behaviour of street-dwellers in Dhaka, Bangladesh. Health Policy Plan. 2009;24:385-94.

46. Omar $\mathrm{H}$, Ibraheem $\mathrm{M}$, Najoua A. Prevalence of intestinal parasite infections among patients in local public hospitals of hail, northwestern Saudi Arabia. Asian Pac J Trop Med. 2016;9(1):44-8.

\section{Publisher's Note}

Springer Nature remains neutral with regard to jurisdictional claims in published maps and institutional affiliations. 